\title{
Riccati Generalized Resonant States of Higher-order Nonlinear Schrödinger Equation With Pöschl-teller Potential
}

Nisha Kadian

Panjab University

Sanjana Bhatia

Panjab University

Shailza Pathania

Panjab University

Amit Goyal ( $\square$ amit2iitb@gmail.com )

GGDSD: Goswami Ganesh Dutta Sanatan Dharma College https://orcid.org/0000-0001-5681-8862

Nagaraja Kumar Choragudi

Panjab University

\section{Research Article}

Keywords: P囚oschl-Teller potential, Higher-order nonlinear Schr囚odinger equation, Isospectral Hamiltonian approach

Posted Date: September 3rd, 2021

DOI: https://doi.org/10.21203/rs.3.rs-722801/v1

License: (c) (1) This work is licensed under a Creative Commons Attribution 4.0 International License.

Read Full License 


\title{
Riccati generalized resonant states of higher-order nonlinear Schrödinger equation with Pöschl-Teller potential
}

\author{
Nisha · Sanjana Bhatia - Shailza \\ Pathania • Amit Goyal · C.N. Kumar
}

Received: date / Accepted: date

\begin{abstract}
We present resonant state solutions of the higher-order nonlinear Schrödinger model, with Pöschl-Teller (PT) potential, under certain parametric conditions. It is found that the localized solutions can be expressed in terms of the hypergeometric functions $F(a, b, c ; z)$. The dynamics of these resonant states and their control using isospectral Hamiltonian approach is well illustrated for PT potential, which is analytically tractable.
\end{abstract}

Keywords Pöschl-Teller potential · Higher-order nonlinear Schrödinger equation · Isospectral Hamiltonian approach

Mathematics Subject Classification (2000) 35C08 35 Q55 $\cdot 78$ A60

\section{Introduction}

The rapid progress in the fabrication and manipulation of high intensity laser beams has lead to ultra fast communication through optical pulses with wavelengths of the order of femtoseconds [1]. Since the early advent of long-distance communication and optical ultrafast switching devices, there has been intense theoretical and experimental investigation of nonlinear localized structures like spatial and spatiotemporal solitons [2,3], similaritons [4], roguewaves [5], and light bullets (LBs) [6]. The dynamics of the propagation of picosecond pulses in nonlinear media is described by the nonlinear Schrödinger equation (NLSE), in which the interplay of Kerr nonlinearity and group velocity dispersion (GVD)

Nisha · S. Bhatia · S. Pathania · C.N. Kumar Department of Physics, Panjab University, Chandigarh 160014, India

A. Goyal

Department of Physics,

Goswami Ganesh Dutta Sanatan Dharma College,

Chandigarh 160030, India

E-mail: amit2iitb@gmail.com, amit.goyal@ggdsd.ac.in 
gives rise to robust localized waves referred to as optical solitons [7]. As the wavelength reduces to $100 \mathrm{fm}$, the higher order nonlinear effects like third order dispersion (TOD), self-steepening (SS) and self-frequency shift (SFS) play a significant role in the pulse dynamics [1]. The propagation of such ultrashort pulses is described by modified NLSE, referred to as higher order-NLSE (HNLSE). The intensity dependent group velocity induces an asymmetry in the already dispersed (due to GVD) pulse [8]. The SS causes the peak of the pulse travelling in the fiber to lean towards the trailing edge leading the latter to become more steeper [9]. For femtosecond pulses, Raman effect can amplify the low-frequency of the broadened spectra, by transferring energy from highfrequency components of the same pulse. This intrapulse energy scattering manifests itself in the form of red-shift of the pulse spectrum as it propagates inside the fiber. This phenomenon is referred to as soliton SFS [10] and is the most dominant among the higher order effects.

Tappert and Zabusky for the first time study the propagation of soliton through inhomogeneous media [11]. In the late $80 \mathrm{~s}$, it was discovered that NLSE in a spatially linear [12] and quadratic potential [13] admits one-soliton solutions that behave exactly like a classical particle. Later on, Knotop et al. [14] investigated the effect of spatio-temporal forces on the dynamics of one-soliton and multi-soliton. In 1994, Nogami and Toyama considered the dynamics of solitons in potential of the type: $V(x, t)=f_{1}(t) x+f_{2}(t) x^{2}$. The modulated beams in nonlinear optics and Bose-Einstein Condensates (BECs) in time dependent magnetic trap can be modelled by using NLSE with time dependent external potential. Serkin et al. [15] explored the non-autonomous solitons for NLSE with linear and harmonic oscillator potential that are a function of both time and spatial coordinate. In 2008, Belmonte-Beitia et al. [16] considered NLSE with nonlinearities and potential that depend both on time and space, reporting nontrivial solutions like breathers, resonant and quasiperiodic solitary waves. Authors have also reported soliton solutions for the NLSE with inhomogeneous complex potential exhibits parity-time symmetry $[17,18]$. A wide class of exact solutions were reported for externally driven Gross-Pitaevskii equation (GPE) in an experimentally generated space-time dependent Jacobian elliptic potential [19]. Because of the variation in the lattice parameters, fiber characteristics are modified. Such systems are described by variable-coefficient NLSE. In recent years, a significant work has been done on the existence of solitons for the NLSE in inhomogeneous media [20-22]. In Ref. [23], the authors have studied the generalised HNLSE in the presence of gravity-like potential to draw parallel between nonlinear solitons and the effects of dark energy and antigravitation effects. In the same year, Youssoufa et al. [24] explored the HNLSE with dual power-law nonlinearities and external potential that can depend on space and time to obtain a family of solitonlike solutions: the so-called W-shaped, dark and the grey solitons. Apart from it, nonautonomous multi-peak solitons [25] and periodic soliton interactions [26] has been studied for HNLSE in inhomogeneous media. In a recent work, the authors have reported the Airy, periodic and Gaussian beams for HNLSE model supported by quadratic, linear and constant potentials, respectively and 
found that chirp associated with these beams can be modulated through SS and SFS parameters. [27].

In this letter, we consider HNLSE with SS and SFS in the presence of Pöschl-Teller (PT) potential [28]. PT potential is exactly solvable and is reflectionless for integral values of the free parameter in the potential. Recently, the stability properties of the exact solutions NLSE in the real PT potential, which is a supersymmetry (SUSY) partner of a complex potential, were investigated [29]. The purpose of this paper is two-fold. First, we attempt to study solution of the HNLSE with PT potential by ansatz method. Undoubtedly, this study will provide a good reference to interpret theoretically the optical waveguide system with the PT potential for the short-range optical communication systems. Second, the so-obtained eigenvalues are used to obtain generalized PT potential and generalised soliton solution for different values of Riccati parameter by using the isospectral Hamiltonian approach.

\section{Model equation}

The HNLSE has been proposed by Kodama and Hasegawa [30] to describe the propagation of short duration (ultrashort or femtosecond) pulses in optical fibers. In Ref. [31], the authors have reported the explicit bright and dark soliton solutions for HNLSE using binary Bell polynomials and auxiliary function methods. In order to model femtosecond pulse propagation, the higher order effects such as the third order dispersion (TOD), the self-steepening, and the self-frequency shift become important if the pulses are shorter than $100 \mathrm{fs}[32,33]$. But for pulses whose width is about $100 \mathrm{fs}$, TOD is normally negligible as compared to the GVD. However, it is very important to take into account the effects of self-steepening and self-frequency shift terms which are still dominant. Under these conditions, we have considered the generalized NLSE (GNLSE) of the form

$$
i Q_{z}+\frac{1}{2} Q_{x x}+|Q|^{2} Q+i \epsilon\left[B\left(|Q|^{2} Q\right)_{x}+C Q\left(|Q|^{2}\right)_{x}\right]-V(x, z) Q=0,
$$

where $Q(x, z)$ is the complex envelope of the electric field, $\epsilon$ indicates over all perturbation parameter and $B$ refers to SS, $C$ signifies SFS term and $V(x, z)$ represents the external potential. Eq. (1) has already been solved to obtained soliton-like solutions with nonlinear chirp $[32,34]$ in the absence of external potential. Recently, we have shown the existence of nonlinear resonant states, in the form of Airy, periodic and Gaussian beams, of the model equation (1) for different choices of potentials $V(x, z)$ [27].

\section{Pöschl-Teller potential}

Let us consider the general Pöschl-Teller (PT) potential,

$$
V(x, z)=\frac{\lambda^{2}-\lambda(\lambda+1) \operatorname{sech}^{2}(\xi)}{2}
$$


where $\xi=(x-v z), v, \lambda$ are real parameters and $V(x, z)$ is governed by parameter $\lambda$ as the depth parameter and was first considered by Epstein [35], and is treated in some quantum mechanics texts. Further, $V(x, z)$ is reflectionless, at any energy, if $\lambda$ is a positive integer. The Schrödinger equation for the PT potential is analytically solvable and its solutions are well known [36].

The PT potential was first introduced by Pöschl \& Teller [28] and by Rosen \& Morse [37]. During the last few decades, a significant work has been done on this potential. Yildirim and Tomak [38] studied several intersubband nonlinear optical properties of this potential. Wang et al. [39] analyzed optical absorption and local-field distribution in a PT quantum well by incorporating nonlocal effects. This potential is a short-range model potential which has been the subject of several studies [40]-[44]. Motivated by these works, here, we explored the nonlinear resonant states for HNLSE with PT potential which governs the ultrashort wave propagation in nonlinear optical media.

\section{Resonant state solutions}

The exact solution to Eq. (1) can be obtained by choosing the ansatz

$$
Q(x, z)=\rho(\xi) e^{i(\psi(\xi)-\omega z)},
$$

where $\xi=(x-v z)$ with $v, \omega$ are real parameters. The functions $\psi(\xi)$ and $\rho(\xi)$ represents the phase and amplitude, respectively. Substitute Eq. (3) into Eq. (1) and solve it to obtain the real and imaginary parts as

$$
\begin{gathered}
v \psi^{\prime} \rho+\omega \rho+\frac{1}{2}\left(\rho^{\prime \prime}-\psi^{\prime 2} \rho\right)+\rho^{3}-\epsilon B \rho^{3} \psi^{\prime}-V(x, z) \rho=0, \\
-v \rho^{\prime}+\rho^{\prime} \psi^{\prime}+\frac{1}{2} \psi^{\prime \prime} \rho+\epsilon(2 C+B) \rho^{2} \rho^{\prime}=0 .
\end{gathered}
$$

Integrating once Eq. (5) gives

$$
\psi^{\prime}=\frac{I}{\rho^{2}(\xi)}+v+\alpha \rho^{2}(\xi)
$$

where $I$ is a constant of integration and $\alpha=-\frac{\epsilon}{2}(2 C+B)$. To avoid singularity in phase, assume $I=0$ and substitute the expression of $\psi^{\prime}$ into Eq. (4), to obtain

$$
\rho^{\prime \prime}+\left(v^{2}+2 \omega-2 V(\xi)\right) \rho+2(1-B \epsilon v) \rho^{3}-\frac{\epsilon^{2}}{4}(2 C+B)(2 C-3 B) \rho^{5}=0 .
$$

The change in frequency across the pulse at any distance $z$ is termed as frequency chirp which can be obtained as

$$
\delta \omega(z, x)=-\frac{\partial}{\partial x}[\psi(\xi)-\omega z]=-\psi^{\prime}(\xi)=-\left(v+\alpha \rho^{2}\right) .
$$

Here, $v$ and $\alpha$ are the chirp parameters representing constant and nonlinear behavior, respectively. The frequency chirp depends considerably on the exact 
pulse shape and can be efficiently controlled through the parameter $\alpha$ by varying the SS and SFS coefficients. The nonlinear chirp parameter ' $\alpha$ ' will be zero for $2 C=-B$ and the corresponding solutions of Eq. (7) have a trivial phase. Here, in this work, we obtain the exact solution, for the governing model, with non-trivial phase modulation for $\alpha \neq 0$. For a constraint on model coefficients such as $2 C=3 B$ and $v=\frac{1}{\epsilon B}$, Eq. (7) reads

$$
\rho^{\prime \prime}-2 V(\xi) \rho+\left(2 \omega+v^{2}\right) \rho=0 .
$$

Intriguingly, Eq. (9) can be identified as quantum mechanical Schrödinger equation, by recognizing $V(\xi)$ as potential term and $\left(2 \omega_{n}+v^{2}\right)$ as corresponding energy level. Choosing $V(\xi)$ as PT potential, one can obtain a class of solutions, in terms of the hypergeometric functions, for different value of $\lambda$. The general solution $\rho_{n}^{\lambda}$ for Eq. (9) is given as $[45,46]$

$$
\rho_{n}^{\lambda}(\xi)=N(\operatorname{sech} \xi)^{\lambda-n} F\left(-n, 2 \lambda-n+1, \lambda-n+1 ; \frac{1-\tanh \xi}{2}\right),
$$

for $n=0,1,2, \cdots<\lambda$ (positive integer), such that

$$
\omega_{n}=-\left(\frac{(\lambda-n)^{2}+v^{2}-\lambda^{2}}{2}\right),
$$

where $N$ is the normalized factor and $F(a, b, c ; f(\xi))$ is the hypergeometric function. Further, we shall discuss the number of resonant states and corresponding solution for the choice of $\lambda=1,2$ and 3 .

Case (i): For $\lambda=1$, Eq. (9) posses only one resonant state given as

$$
\rho_{0}^{(1)}=\frac{1}{\sqrt{2}} \operatorname{sech} \xi,
$$

with constraint condition $\omega_{0}^{(1)}=-\frac{v^{2}}{2}$. Substituting Eq. (12) in Eq. (3), one can obtain the complex wave solution $Q_{0}^{(1)}$ of Eq. (1). In Fig. 1, we have shown the intensity profile corresponding to localized solution $Q_{0}^{(1)}$.

Case (ii): For $\lambda=2, \rho_{n}^{\lambda}$ and corresponding $\omega_{n}$ are given in Table 1. Here, we get nonlinear modes corresponding to different values of $n$. Substituting $\rho_{n}^{\lambda}$ from Table 1 in Eq. (3), the complex wave solution for Eq. (1) can be written as

$$
Q_{n}^{(2)}(x, z)=\rho_{n}^{(2)} e^{i\left(\psi(\xi)-\omega_{n} z\right)},
$$

and the phase profile can be derived using Eq. (6). We have shown the intensity profile for the resonant states, $Q_{0}^{(2)}$ and $Q_{1}^{(2)}$ in Fig. 2. It is found that different modes possess distinct magnitude and number of peaks in the intensity distribution. 


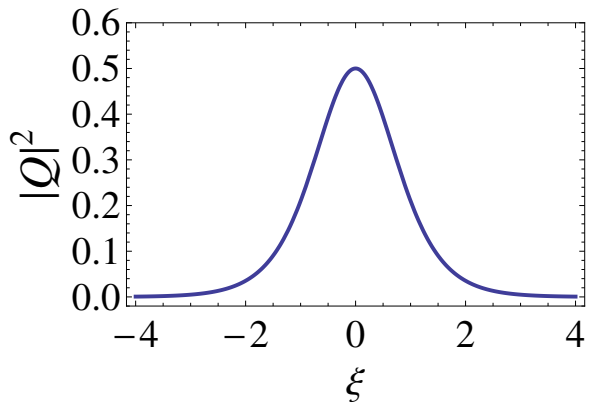

(a)

Fig. 1 The intensity profile of resonant state $Q_{0}^{(1)}$ having the Pöschl-Teller confining potential.

\begin{tabular}{|c|c|c|c|}
\hline$\lambda$ & $n$ & $\rho_{n}^{\lambda}$ & $\omega_{n}$ \\
\hline$\lambda=2$ & $n=0$ & $\rho_{0}^{(2)}=\frac{\sqrt{3}}{2} \operatorname{sech}^{2} \xi$ & $-\frac{v^{2}}{2}$ \\
& $n=1$ & $\rho_{1}^{(2)}=\sqrt{\frac{3}{2}} \operatorname{sech} \xi \tanh \xi$ & $-\frac{\left(v^{2}-3\right)}{2}$ \\
\hline
\end{tabular}

Table 1 The explicit form of resonant states corresponding to $\lambda=2$ for $n=0,1$.

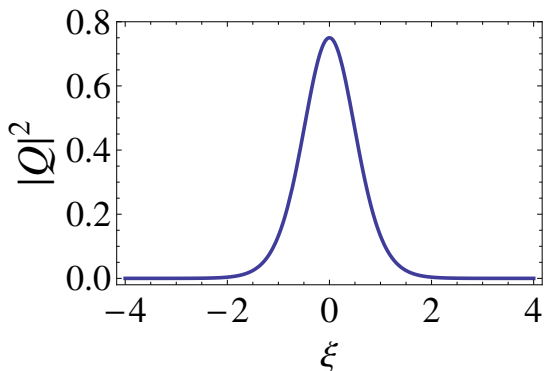

(a)

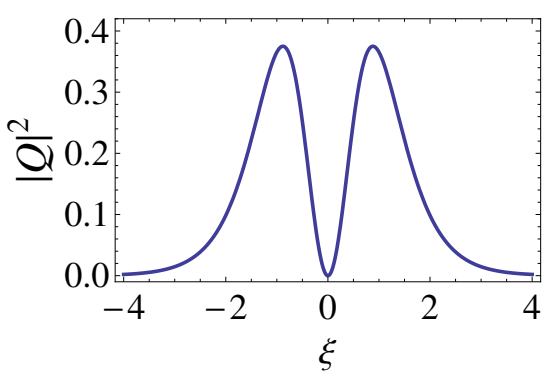

(b)

Fig. 2 The intensity profile of resonant states for the Pöschl-Teller confining potential $(\lambda=2)(\mathrm{a}) Q_{0}^{(2)}$, and (b) $Q_{1}^{(2)}$.

Case (iii): For $\lambda=3$, three resonant states exist for the given potential as given in Table 2. Substituting $\rho_{n}^{\lambda}$ from Table 2 in Eq. (3), the complex wave solution for Eq. (1) can be written as

$$
Q_{n}^{(3)}(x, z)=\rho_{n}^{(3)} e^{i\left(\psi(\xi)-\omega_{n} z\right)},
$$

and the corresponding phase profile can be found from Eq. (6). In Fig. 3, we presented the intensity profile for the resonant states, $Q_{0}^{(3)}, Q_{1}^{(3)}$ and $Q_{2}^{(3)}$, . 
Special Case: For $\lambda=1 / 2$, it can be directly found from Eq. (7) that the nodeless resonate state solution is of the form

$$
\rho_{0}^{\left(\frac{1}{2}\right)}=\operatorname{sech}^{\frac{1}{2}} \xi, \quad \omega_{0}^{(1)}=-\frac{v^{2}}{2}
$$

\begin{tabular}{|c|c|c|c|}
\hline$\lambda$ & $n$ & $\rho_{n}^{\lambda}$ & $\omega_{n}$ \\
\hline$\lambda=3$ & $n=0$ & $\rho_{0}^{(3)}=\frac{\sqrt{15}}{4} \operatorname{sech}^{3} \xi$ & $-\frac{v^{2}}{2}$ \\
& $n=1$ & $\rho_{1}^{(3)}=\sqrt{\frac{15}{4}} \operatorname{sech}^{2} \xi \tanh \xi$ & $-\frac{\left(v^{2}-5\right)}{2}$ \\
& $n=2$ & $\rho_{2}^{(3)}=\frac{\sqrt{3}}{4} \operatorname{sech} \xi\left(-1+5 \tanh ^{2} \xi\right)$ & $-\frac{\left(v^{2}-8\right)}{2}$ \\
\hline
\end{tabular}

Table 2 The explicit form of resonant states corresponding to $\lambda=3$ for $n=0,1,2$.

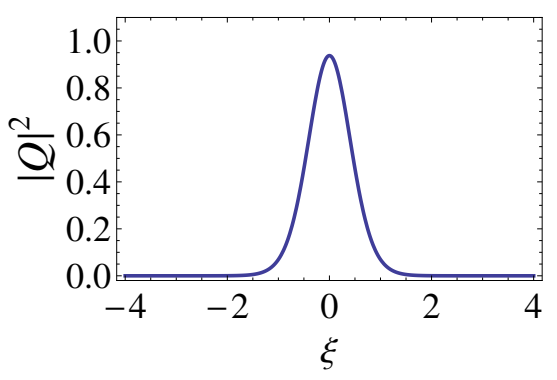

(a)

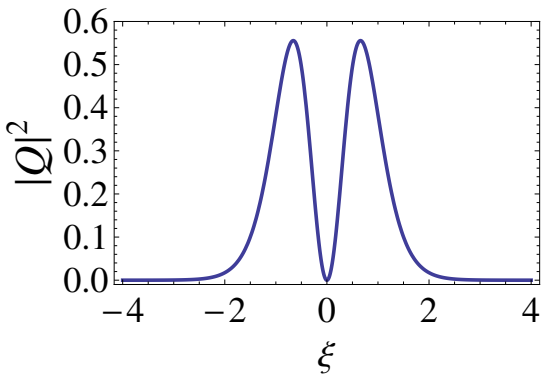

(b)

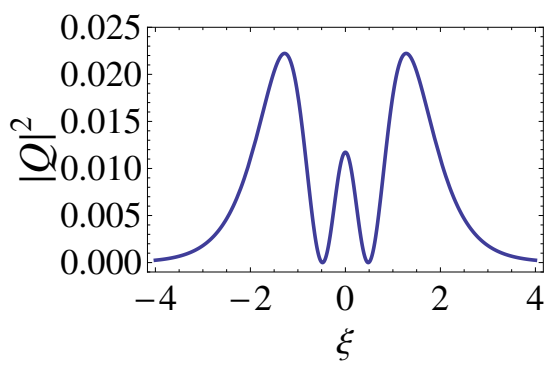

(c)

Fig. 3 The intensity profile of resonant states for the Pöschl-Teller confining potential $(\lambda=3)$ (a) $Q_{0}^{(3)}$, (b) $Q_{1}^{(3)}$, and (c) $Q_{2}^{(3)}$. 


\section{Isospectral Hamiltonian approach-generalized solutions}

An intriguing idea that was first introduced in the field of high-energy physics but has recently found applications in the context of optics is that of SUSY. The advent of SUSY triggered a great interest in the determination of isospectral potentials i.e. potentials which have same eigenvalues, reflection and transmission coefficient. The isospectral Hamiltonian technique is a powerful tool which allows to construct an $n$-parameter family of isospectral potentials from a given one-dimensional potential with $n$-bound states by introducing the Riccati parameter $c[47,48]$. The relationship between the eigenvalues and eigenfunctions of supersymmetric partner potentials is derived and a class of reflectionless potential $\widehat{V}(\xi, c)$ are explicitly constructed which are strictly isospectral to $V(\xi)$. This approach is found useful to generalize and analytically control the dynamical behavior of localized solutions in different physical contexts [49-52].

For the present case, we restrict ourselves to $\lambda=2$, for which Eq. (9) posses two bound state as shown in table 1. To elucidate this discussion, it may be worthwhile to explicitly construct the one-parameter family of strictly isospectral potentials corresponding to the PT potential. The general form of potential and soliton solution is given in Eq. (2) and Eq. (13). For $n=0$, Eq. (2) and Eq. (10) yield

$$
V(\xi)=\frac{1}{2}\left(4-6 \operatorname{sech}^{2} \xi\right), \quad \rho_{0}^{(2)}(\xi)=\frac{\sqrt{3}}{2} \operatorname{sech}^{2} \xi .
$$

The quantity $W(\xi)$ is generally referred to as the superpotential in SUSY quantum mechanics literature [48], defined as

$$
W(\xi)=\frac{-1}{\sqrt{2}} \frac{\rho_{0}^{\prime(2)}(\xi)}{\rho_{0}^{(2)}(\xi)}=\sqrt{2} \tanh (\xi),
$$

and the most general form of $W(\xi)$ can be read as

$$
\widehat{W}(\xi, c)=W(\xi)+\frac{d}{d \xi} \ln \left(c+\int_{-\infty}^{\xi}\left(\rho_{0}^{(2)}(s)\right)^{2} d s\right),
$$

For one parameter deformation, the deformed potential $\widehat{V}(\xi)$ and ground state wave function $\widehat{\rho}_{0}(\xi)$ are obtained as,

$$
\widehat{V}(\xi, c)=V(\xi)-\frac{d}{d \xi}\left(\frac{\left(\rho_{0}^{(2)}(\xi)\right)^{2}}{c+\int_{-\infty}^{\xi}\left(\rho_{0}^{(2)}(s)\right)^{2} d s}\right),
$$

and

$$
\widehat{\rho}_{0}(\xi)=\frac{\sqrt{c(c+1)} \rho_{0}^{(2)}(\xi)}{c+\int_{-\infty}^{\xi}\left(\rho_{0}^{(2)}(s)\right)^{2} d s} .
$$


The deformed excited state wave function for the above potential is obtained as [53],

$$
\widehat{\rho}_{n+1}(\xi, c)=\left(\frac{-d}{d \xi}+\widehat{W}(\xi, c)\right) \phi_{n}(\xi)
$$

Here, $\phi_{n}(\xi)$ is the wavefunction of SUSY partner Hamiltonian isospectral to original Hamiltonian and given as

$$
\phi_{n}(\xi)=\frac{1}{\sqrt{2 \omega_{n+1}+v^{2}}}\left(\frac{d}{d \xi}+W(\xi)\right) \rho_{n+1}(\xi) .
$$

In Fig. 4, we demonstrate the effect of Riccati parameter ' $c$ ' on the partner SUSY potential $\widehat{V}(\xi)$ as given by Eq. (19). For $c=0.01,0.1$ curve corresponds to asymmetric PT potential and for $c=10$ curve represents symmetric PT potential. Thus, it can be concluded that for large values of $c$, profile of $\widehat{V}(\xi) \rightarrow$ $V(\xi)$, although eigen value spectrum always remains same to PT potential.

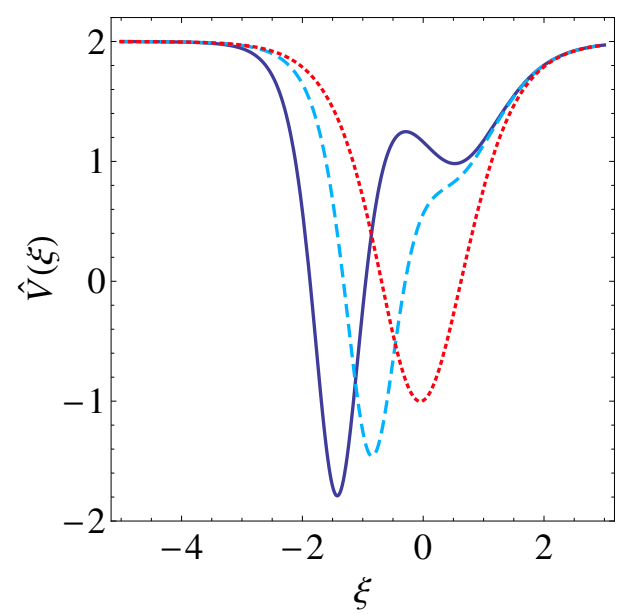

(a)

Fig. 4 Plot of the generalized Pöschl-Teller potential for different values of Riccati parameter $c, c=0.01$ (thick line), $c=0.1$ (dashed line) and $c=10$ (dotted line).

To show the effect of $c$ on the intensity of resonant states for $\lambda=2$, we have plotted the generalized soliton intensity, given by Eq. (3) using Eq. (20) and Eq. (21), for different values of Riccati parameter $c$ in Figs. 5(a) and $5(\mathrm{~b})$, respectively. We find that the intensity for state $Q_{0}^{(2)}$ is larger for smaller values of $c$, and for the large values it approaches the intensity profile without the Riccati generalization, as seen in Fig. 2(a). We also observe that the peaks of the intensity profiles get shifted towards the negative $\xi$ axis for the small positive values of $c$ for $Q_{0}^{(2)}$. A close observation reveal that for small value of Riccati parameter the generalized intensity profile for $Q_{1}^{(2)}$ is asymmetric 


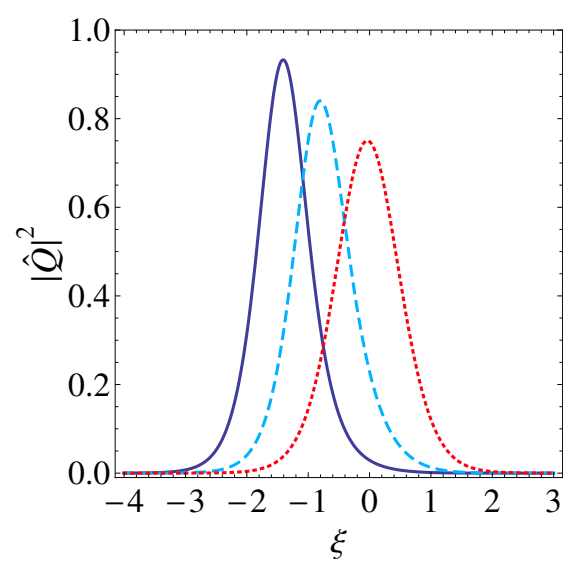

(a)

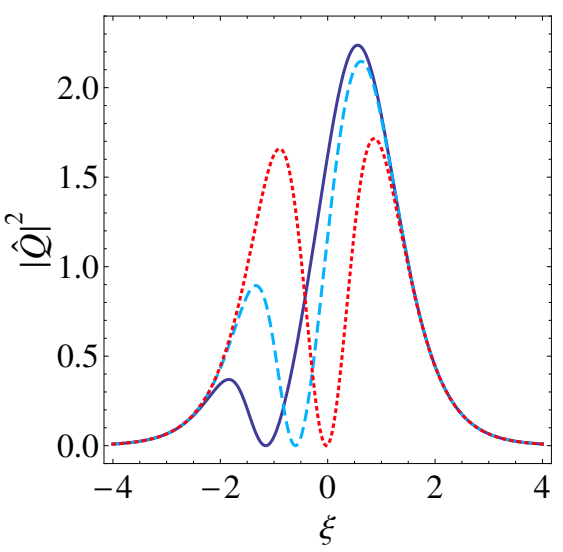

(b)

Fig. 5 Intensity profiles for deformed (generalized) (a) ground state $\widehat{Q}_{0}^{(2)}$ and (b) excited state $\widehat{Q}_{1}^{(2)}$, for different values of Riccati parameter $c, c=0.01$ (thick line), $c=0.1$ (dashed line) and $c=10$ (dotted line).

and energy is distributed between the humps and for large value of the Riccati parameter, it approaches a symmetric profile as is given in Fig. 2(b).

\section{Conclusion}

In this article, a detailed study of resonant states in a higher-order NLSE which models the femtosecond pulse propagation in optical fiber, has been performed. Our interest in these Pöschl-Teller resonant states mostly lies in fact that the free parameter in PT potential imposes significant effects on the intensity of optical beams. These nonlinear resonant states are analytically illustrated in terms of Gaussian beams that resulted due to the presence of Pöschl-Teller potential of the ensuing model. We have determined the nonlinear chirp associated with these solitary waves which can be efficiently tuned through self-steepening and self-frequency shift parameters.

Further, employing the isospectral deformation technique, we have worked out the deformed ground state and first excited state solution for $\lambda=2$ with a corresponding deformed potential through the introduction of Riccati parameter $c$. It is found that even small variations in the Riccati parameter lead to rapid shift in intensity profiles. It has been found that for suitably chosen parameter values number of resonant states with constraints can be found for various choice of the system parameters. 


\section{Acknowledgments}

A.G. and Nisha would like to thank Science and Engineering Research Board (SERB), Government of India for the award of SERB Start-Up Research Grant (Young Scientists) and student fellowship, respectively, under the sanction no: YSS/2015/001803, during the course of this work. S.B. and S.P. would like to thank Department of Science and Technology (DST), Government of India for Junior Research Fellowship through Inspire Scheme under the sanction no: IF190338 and IF170725, respectively.

\section{Compliance with ethical standards}

\section{Conflict of interest}

The authors declare that they have no conflict of interest.

\section{Data availability}

Data sharing not applicable to this article as no datasets were generated or analysed during the current study.

\section{References}

1. Agrawal, G.P.: Nonlinear Fiber Optics, Academic Press, San Diego (2007)

2. Bjorkholm, J.E., Ashkin, A.A.: cw Self-Focusing and Self-Trapping of Light in Sodium Vapor. Phys. Rev. Lett. 32, 129-132 (1974)

3. Zhong, W., Belić, M.R., Huang, T.: Solitary waves in the nonlinear Schrödinger equation with spatially modulated Bessel nonlinearity. J. Opt. Soc. Am. B 30, 1276-1283 (2013)

4. Dai, C., Wang, Y., Zhang, J.: Analytical spatiotemporal localizations for the generalized $(3+1)$-dimensional nonlinear Schrödinger equation. Opt. Lett. 35, 1437-1439 (2010)

5. Solli, D.R., Ropers, C., Koonath, P., Jalali, B.: Optical rogue waves. Nature 450, 10541057 (2007)

6. Chen, S., Dudley, J.M.: Spatiotemporal Nonlinear Optical Self-Similarity in Three Dimensions. Phys. Rev. Lett. 102, 233903 (2009)

7. Hasegawa, A., Tappert, F.: Transmission of stationary nonlinear optical pulses in dispersive dielectric fibers. Appl. Phys. Lett. 23, 142-144 (1973)

8. Shen, S., Chang, C., Sardesai, H.P., Binjrajka, V., Weiner, A.M.: Effects of self-phase modulation on sub-500 fs pulse transmission over dispersion compensated fiber links. J. Lightwave Technol. 17, 452-461 (1999)

9. Fork, R.L., Shank, C.V., Hirlimann, C., Yen, R., Tomlinson, W.J.: Femtosecond whitelight continuum pulses. Opt. Lett. 8, 1-3 (1983)

10. Mitschke, F.M., Mollenauer, L.F.: Discovery of the soliton self-frequency shift. Opt. Lett. 11, 659-661 (1986)

11. Tappert, F.D., Zabusky, N.J.: Gradient-Induced Fission of Solitons. Phys. Rev. Lett. 27, 1774-1776 (1971)

12. Chen, H.H., Liu, C.S.: Solitons in Nonuniform Media. Phys. Rev. Lett. 37, 693-697 (1976)

13. Konotop, V.V.: Soliton on a disordered lattice. Phys. Rev. E 47, 1423-1426 (1993)

14. Nogami, Y., Toyama, F.M.: Nonlinear Schrödinger soliton in a time-dependent quadratic potential. Phys. Rev. E 49, 4497-4501 (1994) 
15. Serkin, V.N., Hasegawa, A., Belyaeva, T.L.: Nonautonomous Solitons in External Potentials. Phys. Rev. Lett. 98, 074102 (2007)

16. Belmonte-Beitia, J., Pérez-García, V.M., Vekslerchik, V., Konotop, V.V.: Localized Nonlinear Waves in Systems with Time- and Space-Modulated Nonlinearities. Phys. Rev. Lett. 100, 164102 (2008)

17. Wang, Y.Y., Dai, C.Q., Wang, X.G.: Stable localized spatial solitons in PT-symmetric potentials with power-law nonlinearity. Nonlinear Dyn. 77, 1323-1330 (2014); Dai, C.Q. Fan, Y., Wang, Y.Y.: Three-dimensional optical solitons formed by the balance between different-order nonlinearities and high-order dispersion/diffraction in parity-time symmetric potentials. Nonlinear Dyn. 98, 489-499 (2019)

18. Hanif, Y., Sarfraz, H., Saleem, U., Dynamics of loop soliton solutions of PT-symmetric nonlocal short pulse equation, Nonlinear Dyn. 100, 1559-1569 (2020)

19. Raju, T.S., Panigrahi, P.K.: Exact solutions of the modified Gross-Pitaevskii equation in 'smart' periodic potentials in the presence of external source. J. Nonl. Math. Phys. 18, 367-376 (2011)

20. Zhang, Y., Yang, C., Yu, W., Mirzazadeh, M., Zhou, Q., Liu, W.: Interactions of vector anti-dark solitons for the coupled nonlinear Schrödinger equation in inhomogeneous fibers. Nonlinear Dyn. 94, 1351-1360 (2018)

21. Kengne, E., Lakhssassi, A., Liu, W.M.: Non-autonomous solitons in inhomogeneous nonlinear media with distributed dispersion. Nonlinear Dyn. 97, 449-469 (2019)

22. Sulaiman, T.A., Yusuf, A., Alquran, M.: Dynamics of optical solitons and nonautonomous complex wave solutions to the nonlinear Schrödinger equation with variable coefficients. Nonlinear Dyn. 104, 639-648 (2021)

23. Serkin, V.N., Belyaeva, T.L.: Nontrivial Galilean-like invariance of the generalized higher-order nonlinear Schrödinger equation model with gravitation-like potential and the soliton analogies of the cosmic dark energy and antigravitation effects. Optik 160, 389-395 (2018)

24. Youssoufa, M., Dafounansou, O., Mohamadou, A.: W-shaped, dark and grey solitary waves in the nonlinear Schrödinger equation competing dual power-law nonlinear terms and potentials modulated in time and space. J. Mod. Opt. 66, 530-540 (2019)

25. Cai, L.Y., Wang, X., Wang, L., Li, M., Liu, Y., Shi, Y.Y.: Nonautonomous multipeak solitons and modulation instability for a variable-coefficient nonlinear Schrödinger equation with higher-order effects. Nonlinear Dyn. 90, 2221-2230 (2017)

26. Chen, J., Luan, Z., Zhou, Q., Alzahrani, A.K., Biswas, A., Liu, W.: Periodic soliton interactions for higher-order nonlinear Schrödinger equation in optical fibers. Nonlinear Dyn. 100, 2817-2821 (2020)

27. Pathania, S., Goyal, A., Raja, T.S., Kumar, C.N.: Chirped nonlinear resonant states in femtosecond fiber optics. Optik 227, 166094 (2021)

28. Pöschl, G., Teller, E.: Bemerkungen zur Quantenmechanik des anharmonischen Oszillators. Zeitschrift für Physik 83, 143-151 (1933)

29. Cooper, F., Khare, A., Comech, A., Mihaila, B., Dawson, J.F., Saxena, A.: Multiple slow waves in metaporous layers for broadband sound absorption. J. Phys. A 50, 015301 (2017)

30. Kodama, Y., Stat., J.:Optical solitons in a monomode fiber. Phys. 39, 597-614 (1985)

31. Li, M., Xu, T., Wang, L.: Dynamical behaviors and soliton solutions of a generalized higher-order nonlinear Schrödinger equation in optical fibers. Nonlinear Dyn. 80, 1451-1461 (2015)

32. Vyas, V.M., Patel, P., Panigrahi, P.K., Kumar, C.N., Greiner, W.: Chirped chiral solitons in the nonlinear Schrödinger equation with self-steepening and self-frequency shift. Phys. Rev. A 78, 021803(R) (2008).

33. Alka, Goyal, A., Gupta, R., Kumar, C.N., Raju, T.S.: Chirped femtosecond solitons and double-kink solitons in the cubic-quintic nonlinear Schrödinger equation with selfsteepening and self-frequency shift. Phys. Rev. A 84, 063830 (2011)

34. Kumar, C.N., Durganandini, P.: New phase modulated solutions for a higher-order nonlinear Schrdöinger equation. Pramana J. Phys. 53, 271-277 (1999)

35. Epstein, P.S.: Reflection of waves in an inhomogeneous absorbing medium. Proc. Natl. Acad. Sci. U.S.A. 16, 627-637 (1930)

36. Flugge, S.: Practical Quantum Mechanics-I, Springer-Verlag, Berlin (1971) 
37. Rosen, N., Morse, P.M.: On the Vibrations of Polyatomic Molecules. Phys. Rev. 42, 210-217 (1932)

38. Yildirim, H., Tomak, M.: Nonlinear optical properties of a Pöschl-Teller quantum well. Phys. Rev. B 72, 115340 (2005)

39. Wang, G., Guo, Q., Wu, L., Yang, X.: Optical response and local-field distribution in a Pöschl-Teller quantum well: Nonlocal effects. Phys. Rev. B 75, 205337 (2007)

40. Dong, S.H., Gonzalez-Cisneros, A.: Energy spectra of the hyperbolic and second Pöschl-Teller like potentials solved by new exact quantization rule. Ann. Phys. 323, 1136-1149 (2008)

41. Unal, V.U., Aksahin, E., Aytekin, O.: Electric field effect on the refractive index changes in a Modified-Pöschl-Teller quantum well. Phys. Rev. E 47, 103-108 (2013)

42. Zhang, Z.H., Liu, C., Guo, K.X.: Electron-phonon interaction effect on the refractive index changes in a Modified-Pöschl-Teller quantum well. Optik 127, 1590-1594 (2016)

43. Shea, P., van Zyl, B.P., Bhaduri, R.K.: The two-body problem of ultra-cold atoms in a harmonic trap. Am. J. Phys 77, 511-515 (2009)

44. Goswami, P., Deb, B.: A model study on a pair of trapped particles interacting with an arbitrary effective range. Phys. Scr. 91, 085401 (2016)

45. Morse, P.M., Feshbach, H.: Methods of Theoretical Physics, McGraw-Hill, New York (1953)

46. Dong, S.H., Lemus, R.: Ladder Operators for the Modified Pöschl-Teller Potential. Int. J. Quantum Chem. 86, 265-272 (2002)

47. Mielnik, B.: Factorization method and new potentials with the oscillator spectrum. J. Math. Phys. 25, 3387-3389 (1984)

48. Cooper, F., Khare, A., Sukhatme, U.: Supersymmetry and quantum mechanics. Phys. Rep. 251, 267-385 (1995)

49. Kumar, C.N.: Isospectral Hamiltonians: generation of the soliton profile. J. Phys. A: Math. Gen. 20, 5397-5401 (1987)

50. Drigo Filho, E., Ruggiero, J.R.: H-bond simulation in DNA using a harmonic oscillator isospectral potential. Phys. Rev. E 56, 4486-4488 (1997); Alka, W., Goyal, A., Kumar, C.N.: Nonlinear dynamics of DNA - Riccati generalized solitary wave solutions. Phys. Lett. A 375, 480-483 (2011)

51. Kumar, C.N., Gupta, R., Goyal, A., Loomba, S., Raju, T.S., Panigrahi, P.K.: Controlled giant rogue waves in nonlinear fiber optics. Phys. Rev. A 86, 025802 (2012); De, K.K., Goyal, A., Raju, T.S., Kumar, C.N., Panigrahi, P.K.: Riccati parameterized self-similar waves in two-dimensional graded-index waveguide. Opt. Commun. 341, 15-21 (2015)

52. Panigrahi, P.K., Gupta, R., Goyal, A., Kumar, C.N.: Riccati generalization of self-similar solutions of nonautonomous Gross-Pitaevskii equation. Eur. Phys. J. Spec. Top. 222, $655-663$ (2013)

53. Khare, A.: Supersymmetry in quantum mechanics. AIP Conf. Proc. 744, 133 (2005) 